\title{
The impact of dizziness on quality-of-life in the elderly
}

\author{
Andrea Ciorba $^{1} \cdot$ Chiara Bianchini $^{1} \cdot$ Giovanni Scanelli $^{2} \cdot$ Marco Pala $^{3}$. \\ Amedeo Zurlo ${ }^{3}$ - Claudia Aimoni ${ }^{1}$
}

Received: 10 June 2016/ Accepted: 18 July 2016

(C) Springer-Verlag Berlin Heidelberg 2016

\begin{abstract}
Dizziness is a common medical condition that has been related to falls in the elderly, and it is, therefore, considered a severe social health problem. Particularly in the elderly, the impact of dizziness may be relevant, as it has been linked to several conditions, such as isolation, depression, reduced self autonomy, and self control. The social, functional, and psychological well-being of those affected can be hampered significantly, thus reducing the quality-of-life (QoL) perception. In addition, due to the aging of the population in the developed world, dizziness is becoming a growing public health problem; an optimal management of this condition includes, nowadays, the improvement of rehabilitative programs, as well as the evaluation of QoL status and its management. The aim of this paper is to evaluate the impact of dizziness on the QoL in the elderly, also analyzing the instruments available, nowadays, to evaluate QoL of dizzy patients.
\end{abstract}

Keywords Dizziness - Presbystasis - Risk of falls · Elderly · Older adults · Quality-of-life

Andrea Ciorba

andrea.ciorba@unife.it

1 ENT and Audiology Department, S. Anna University Hospital of Ferrara, Via Aldo Moro, 8, 44124 Ferrara (Cona), Italy

2 Internal Medicine Department, University Hospital of Ferrara, Ferrara, Italy

3 Geriatric Department, University Hospital of Ferrara, Ferrara, Italy

\section{Introduction}

Dizziness is the term used to indicate a condition that affects individual's balance; since one of the most serious consequences of dizziness is falls, it represents a growing public health problem in the elderly. The increased susceptibility to falling can significantly hamper the qualityof-life perception [1-3], as many elderly who fall develop a fear of falling that consequently affects their daily activities.

Dizziness can depend on many factors, including cardiovascular/neurological disease, sensorial deterioration (i.e., visual deficits), blood pressure imbalance, and vestibular dysfunction, as each task that is related to the maintenance and control of equilibrium deteriorates with aging (Table 1) [1, 3, 4]. Therefore, this is a challenging condition for physicians, both in terms of diagnostic and therapeutic approach, due to the wide range of underlying conditions [1-3].

A correct management to this condition should include a proper diagnostic assessment, the development of rehabilitative strategy/strategies, as well as the evaluation of quality-of-life (QoL) status and its management.

The aim of the present review is: (1) to analyze the instruments that have been proposed to assess QoL of the dizzy patient, and, therefore, (2) to evaluate the impact of dizziness on QoL in the elderly.

\section{Methods}

A literature review was planned and performed using different databases: Medline/PubMed, EMBASE, and CINAHL, also according to PRISMA guidelines. All databases have been searched from 2005 up to December 
Table 1 Possible etiology of dizziness in the elderly [1-28]

\begin{tabular}{ll}
\hline Vestibular & Vestibular neuritis \\
disorders & Meniere disease \\
& Bening paroxysmal positional vertigo (BPPV) \\
& Vestibular schwannoma (conservatively \\
managed) & Ototoxicity \\
Non-vestibular & Central nervous system disorders \\
disorders & Transient ischaemic attack \\
& Stroke \\
& Neurodegenerative disorders \\
& Musuloskeletal system disorders \\
& Osteoarthritis \\
& Microcrystal disorders \\
& Paget's disease \\
& Cardiovascular diseases \\
& Hypotension \\
& Blood pressure imbalance \\
Myocardial infarction & Arrhythmia \\
Visual defects & Vision loss \\
Cataracts \\
Medications (i.e., antihypertensive agents, \\
anticonvulsivants, anxiolytics, \\
antidepressants, antipsychotics) \\
\\
\\
\end{tabular}

2015. The medical subject heading (MeSH) terms used for the search included: dizziness, elderly, older adults, falls, and quality-of-life. Other terms also searched were: presbystasis and risk of falls. Full-text articles were obtained in cases, where the title, abstract, or key words suggested that the study may be eligible for this review.

The search was carried out independently by two of the authors (A.C. and C.B.), and restricted to papers in English. The initial search returned a total of 149 articles. Other papers $(n=18)$ were also identified from a further manual check of references included in the published literature. Inclusion criteria were: age $>65$ years, clinical series, and review papers. Exclusion criteria were: full text not available; manuscript not in English; case reports, paper already identified using one of the above databases, and studies emphasizing other topics, such as presbystasis or tinnitus. The authors, subsequently, met to critically discuss disagreements on citation inclusions. A total of 54 papers were then excluded, according to both the authors (A.C. and C.B.) (see also Table 2).

Subsequently, we have performed a critical appraisal of the 113 selected papers, by reading abstracts and/or texts, to decide whether the identified papers were relevant to this search or not. In particular, inclusion criteria were: for clinical series, papers with an adequate group of patients
Table 2 Papers resulted from the search through years 2005-2015

Total number of articles obtained after the PubMed search 71

Total number of articles obtained after the EMBASE search 52

Total number of articles obtained after the CINAHL search 26

Other papers from references in the published literature 18

Total number of papers identified 167

Papers excluded $^{\mathrm{a}} \quad 54$

Total number of articles assessed for eligibility 113

Further papers excluded $^{\text {b }} \quad 88$

Total number of papers finally included 25

${ }^{a}$ Inclusion criteria were: age $>65$ years, clinical series, review papers. Exclusion criteria were: full text not available, manuscript not in English language, case reports, paper already identified using one of the above databases, and studies emphasizing other topics, such as presbystasis or tinnitus

b Inclusion criteria were: for clinical series, papers with an adequate group of patients studied (at least $n>50$ patients) and with patient assessment also through dizziness-specific QoL instruments; for reviews, papers published on relevant journals and papers showing a rigorous methods and rigorous reporting

studied (at least $n>50$ patients) and with patient assessment also through dizziness-specific QoL instruments; for reviews, papers published on relevant journals and papers showing a rigorous methods and rigorous reporting. Therefore, after a critical evaluation, a total of 25 results were finally identified as appropriate for this study, so were obtained and reviewed in detail by the authors.

\section{Epidemiology and risk factors}

According to recent esteems in the US and in the UK, dizziness is reported to be one of the most common symptoms complained among patients aged $>65$ years. In particular, Lin et al. have estimated that one out of five elderly persons in the US experiences dizziness and/or imbalance in the last life years [1-3]. The frequency of dizziness is reported to increase with age [5] and females are more likely to experience balance problems than males [1-7]. Dros et al have estimated the prevalence of dizziness in about $30 \%$ in those aged over 65 years and in $>50 \%$ in those aged 85 and more [8]. In addition, some reports have indicated that older adults with history of dizziness and imbalance have an higher risk of falling [4, 9-11].

The underlying cause of dizziness in the elderly is complex [4, 12, 13]. General population studies have reported that dizziness of vestibular origin can be estimated in about $15 \%$ of the general population aged $>70$ years; while non-vestibular causes (i.e., cerebrovascular or neurological causes) have been reported to range between 20 and $70 \%$ in dizzy patients aged $>70$ years $[4,12,14]$. 
Dizziness can be episodic or subcontinuous; in both cases, it is reported to affect quality-of-life in the elderly in several ways, as it has been correlated to the onset of negative outcomes, such as depressive statuses, reduced self autonomy, and self control, as well as to increased fear of falling [5]. It can be troublesome for many patients, as it can then worsen social isolation, functional disabilities, and can favorite the need of nursing home placement $[5,8]$.

Some authors have also indicated that the coexistence of other diseases (i.e., anxiety, depressive disorders, as well as the use of sedative drugs) can exacerbate the impact of dizziness on everyday life and, therefore, on QoL perception $[5,8]$.

\section{Instruments to assess the QoL of the dizzy patient}

The term quality-of-life (QoL) is used to define the overall well-being of each subject. According to the literature reports, the evaluation of QoL is multi-dimensional and includes different domains, such as physical, social, and emotional well-being [7, 13, 15].

Several instruments have been proposed to assess QoL of the patient with dizziness, as reported by different studies [1, 5-8, 16-21]. These can be divided into dizziness-specific QoL instruments and generic QoL instruments.

\section{Dizziness-specific QoL tools}

- Dizziness handicap inventory It was developed and validated in 1990 by Jacobson and Newman [17]. This is a self-report questionnaire originally designed to quantify the handicapping effect of dizziness imposed by vestibular system disease, but it has also been used for persons with dizziness of other origins [6]. It has now been validated and is available in different languages; it aims to measure the effects of dizziness on physical, emotional, and functional sub-domains $[6,18-21]$. It is probably the most employed nowadays.

- Vertigo Symptom Scale-short form (VSS-sf) This is a specific questionnaire assessing the severity of vertigo/ dizziness symptoms, by evaluating the frequency of the episodes during the period of time of a month and also the related autonomic symptoms (i.e., nausea, vomiting episodes). It comprises 15 items, 8 for the impact of dizziness, and 7 for the autonomic symptoms [6].

- The Disability Scale is a global self-report measure, and it is used to assess disability in connection with dizziness [6]. The scale does not refer to any time period. It is scored on a 6-points ordinal scale, counting from 0 (no disability), to 5 (severe disability) [6].
- The Vertigo Handicap Questionnaire (VHQ) also assess the disabling consequences of vertigo, particular investigating among the recurrence of vertigo episodes. It is composed by a questionnaire of 22 items in total [18]; each item scores from 0 (no handicap) to 4 (maximum handicap) [18].

- The Activities-Specific Balance Confidence scale (ABC scale) is an instrument consisting of 16 items, developed with the aim of investigating among the fear of falling in the older population. It explores the grade of daily activity difficulty (i.e., walking in the house or on icy sidewalks) [18, 19].

- The UCLA Dizziness Questionnaire also evaluates the frequency and severity of vertigo episodes, in a period of time, using five items [18].

- The Vestibular Disorders Activity of Daily Living (VADL) scale allows the patients to evaluate the selfperceived level of discomfort related to dizziness on daily activities (range: $1=$ independent; $10=$ too difficult to perform). It consists of 28 items in total $[18,20,21]$.

\section{Generic QoL measures}

Those do not focus on any particular disorder or treatment, but evaluate the self-perceived, global, health status of the individual. The most commonly administrated, together with dizziness-specific tools, are:

- The Short Form Health Survey (SF-36). This tool consists of 36 items that assesses eight health concepts. In particular, it values limitations in: (1) physical activities due to health problems; (2) social activities, because of physical or emotional problems; (3) in usual role activities due to physical health problems; (4) in usual role activities, because of emotional problems; (5) presence of bodily pain; (6) general mental health (psychological distress and well-being); (7) vitality (energy and fatigue); and (8) general health perceptions [7-30].

- COOP/WONCA is another generic tool measuring health status. It refers to the previous 2 weeks, and includes several charts assessing physical fitness, social activities, changes in health, and overall health [7].

- Hospital Anxiety and Depression Scale (HADS) This is a self-assessment questionnaire used to measure anxiety and depression in a week time-frame. The questionnaire comprises 14 items, 7 for anxiety, and 7 for depression [30].

- The multi-dimensional geriatric assessment (comprehensive geriatric evaluation-CGE) allows to explore the components of subject's welfare, particularly the affective state using specific tools (i.e., Geriatric 
Depression Scale, Hamilton Rating Scale for Depression, and Zung Self Rating Depression Scale) also allowing an objective assessment of patient's functional abilities through specific tools (i.e., ADL, IADL, and Barthel Index) [18-30].

\section{Evaluation of QoL in the dizzy patients: present data}

Understanding the impact of dizziness on QoL is of great importance, as the social and psychological well-being of the elderly person can be hampered significantly by this condition. In addition, patients' self perception of QoL is becoming an increasing recognized indicator for health care evaluation, and the correct management of this condition, therefore, cannot disregard from an adequate $\mathrm{QoL}$ assessment [1, 7, 25].

Several authors have reported that older adults with dizziness show a deteriorated QoL perception, independently from the underlying etiology [28, 29], and, in particular, reported effects of dizziness on QoL are: lowliness, isolation, depression, frustration, decreased self esteem, and loneliness $[8,25,28]$. One of the main aspects that impact the QoL perception is related to the fact that a specific diagnosis of the cause of dizziness often lacks. It has been reported that up to $20-40 \%$ of patients can remain without a specific diagnosis, either because dizziness in the elderly can be multi-causal, either because it is not possible to identify a specific etiologic condition at the end of the diagnostic algorithm. Particularly, the presence of a persistent dizziness status can lead to significant status of psychological distress with impairment of daily functioning, social isolation, and anxiety [8] (see also Table 3).

There are very few studies in the literature investigating among QoL status in patients affected by dizziness due to vestibulopaties. In Meniere disease patients, a lower QoL perception has been described, and it has been associated to isolation, frustration, decreased self esteem, and loneliness [31]. In these cases, QoL has been reported to be more deteriorated when symptoms are more severe or their duration is longer [31]; moreover, vestibular symptoms of Meniere disease have been described as the factors with the greatest impact among QoL perception [32]. In addition, those affected by vestibular schwannoma and benign paroxysmal positional vertigo have been reported to show a lower QoL status perception in respect to non-dizzy subjects of same age $[23,25]$. We could not retrieve studies that have investigated among $\mathrm{QoL}$ status and vestibular neuronitis so far $[8,33]$.

In those affected by dizziness not related to vestibular disorders, anxiety, agoraphobia, fear to fall, and major depression, as well as the perception of a social isolation have been reported to be present in up to $50 \%$ of patients [33]. The QoL of this group of patients has been reported to worse progressively especially when it is difficult to identify the origin and the nature of the dizziness problem and when recurrent episodes and/or unpredictable attacks characterize the clinical story of the patient [33]. Especially in these cases, chronic dizziness can lead to loss of confidence, irritability, fear of going out alone, and patients can then further restrict their physical and social activities to reduce the risk of symptoms occurrence in uncomfortable situations [33, 34]. Investigating among QoL could also help in discriminating what part of the loss of autonomy can be attributed to psychological depressive factors (that can eventually be corrected pharmacological or nonpharmacologically), and which to the chronic dizziness itself.

\section{QoL of the dizzy patients after rehabilitation}

Rehabilitation programs have been introduced by several authors to achieve a better postural stability, especially in multi-factorial dizzy patients aged $>65$ years.

In vestibular defective patients, rehabilitative treatments have been reported to improve QoL perception $[33,34]$. QoL may decrease initially by changing elderly's daily routine and making it more active and exhausting, especially when short training intervention programs have been established [16]. However, QoL has been then reported to improve in long-term rehabilitative settings [16]. In particular, customized vestibular rehabilitation programs (i.e., vestibular rehabilitation in individuals with peripheral vestibular syndrome of
Table 3 Prevalent reported effects of dizziness on QoL, due to vestibular and non-vestibular disorders

\begin{tabular}{ll}
\hline $\begin{array}{l}\text { Effects of dizziness } \\
\text { in vestibular disorders }\end{array}$ & $\begin{array}{l}\text { Effects of dizziness } \\
\text { in non-vestibular disorders }\end{array}$ \\
\hline Lowliness & Anxiety \\
Isolation & Agoraphobia \\
Frustration & Fear to fall \\
Decreased self esteem & Major depression \\
Loneliness & Social isolation \\
\hline
\end{tabular}


labyrinthine origin) have been shown to improve QoL perception independently by gender and age [22]. In addition, patients dizzy by benign paroxysmal positional vertigo and Meniere disease undergoing rehabilitative programs have been showing great improvement of QoL perception on the physical and functional aspects of QoL [23].

Among dizziness not related to vestibular problems, most authors agree that multidisciplinary medical approach and physiotherapy, during diagnosis and treatment, are also necessary to achieve greater security and adherence to treatment modality and, therefore, to improve QoL perception in those subjects $[16,22,23]$. Some authors have demonstrated that improvement in QoL status of subjects with undefined dizziness can be obtained after physical rehabilitation that can improve muscle strength and balance. QoL improvement has been rated between 17 and $46 \%$ [16].

Finally, few authors have investigated among the use of otoneurological and neurological medication; treatments have not been associated to changes in QoL perception [24]. Only betahistine has been reported to have some efficacy in improving the patients' physical, functional and emotional status when treating dizziness patients due to vestibulopaties at the earliest stages. [34].

\section{Conclusions}

As dizzy patients suffer significant QoL impairment, QoL assessment should always integrate the diagnostic assessment of the dizzy patient, especially in the elderly. Indeed, further research is necessary to understand the many factors underlying this condition and, in particular, those impacting health status perception, and, therefore, to give further treatment options to these subjects. Establishing an appropriate approach through rehabilitative programs can effectively reduce patients' disability perception and improve their QoL status [33].

Furthermore, repeated evaluation of QoL can be a proxy indicator of the effectiveness of rehabilitation therapy, and therefore, it can also be used as a therapeutic and prognostic impact indicator during the follow-up of these patients.

\section{Compliance with ethical standards}

This article does not contain any studies with human participants or animals performed by any of the authors.

Funding None to declare.

Conflict of interest None to declare.

\section{References}

1. Ciorba A (2015) Dizziness and the risk of falling in the elderly: a literature review. J Hear Sci 1:9-13

2. Lin HW, Bhattacharyya N (2012) Balance disorders in the elderly: epidemiology and functional impact. Laryngoscope 122:1858-1861

3. Ciorba A, Hatzopoulos S, Bianchini C, Aimoni C, Skarzynski H, Skarzynski PH (2015) Genetics of presbycusis and presbystasis. Int J Immunopathol Pharmacol 28(1):29-35

4. Iwasaki S, Yamasoba T (2014) Dizziness and imbalance in the elderly: age-related decline in the vestibular system. Aging Dis 9:6(1):38-47

5. Ekwall A, Lindberg A, Magnusson M (2009) Dizzy—why not take a walk? Low level physical activity improves quality of life among elderly with dizziness. Gerontology 55:652-659

6. Tamber AL, Wilhelmsen KT, Strand LI (2009) Measurement properties of the Dizziness Handicap Inventory by cross-sectional and longitudinal designs. Health Qual Life Outcomes 7:101

7. Ciorba A, Bianchini C, Pelucchi S, Pastore A (2012) The impact of hearing loss on the quality of life of elderly adults. Clin Interv Aging 7:159-163

8. Dros J, Maarsingh OR, Beem L, van der Horst HE, ter Riet G, Schellevis FG, van Weert HC (2011) Impact of dizziness on everyday life in older primary care patients: a cross-sectional study. Health Qual Life Outcomes 16(9):44

9. O’Loughhlin JL, Boivin JF, Robitaille Y, Suissa S (1994) Falls among the elderly: distinguishing indoor and outdoor risk factors in Canada. J Epidemiol Community Health 48:488-489

10. Rubinstein LZ, Josephson KR (2006) Falls and their prevention in the elderly people: what does the evidence show? Med Clin North Am 90:807-824

11. Stel VS, Pluijm SM, Deeg DJ, Smit JH, Bouter LM, Lips P (2003) A classification tree for predicting recurrent falling in community-dwelling older persons. J Am Geriatric Soc 51:1356-1364

12. Lawson J, Fitzgerald J, Birchall J, Aldren CP, Kenny RA (1999) Diagnosis of geriatric patients with severe dizziness. J Am Geriatr Soc 47(1):12-17

13. Felce D, Perry J (1995) Quality of life: its definition and measurement. Res Dev Disabil 16(1):51-74

14. Colledge NR, Barr-Hamilton RM, Lewis SJ, Sellar RJ, Wilson JA (1996) Evaluation of investigations to diagnose the cause of dizziness in elderly people: a community based controlled study. BMJ 313:788-792

15. Felce D (1997) Defining and applying the concept of quality of life. J Intellect Disabil Res 41(2):126-135

16. Tuunainen E, Rasku J, Jäntti P, Moisio-Vilenius P, Mäkinen E, Toppila E, Pyykkö I (2013) Postural stability and quality of life after guided and self-training among older adults residing in an institutional setting. Clin Interv Aging 8:1237-1246

17. Jacobson GP, Newman CW (1990) The development of the dizziness handicap inventory. Arch Otolaryngol HeadNeck Surg 116(4):424-427

18. Alghwiri AA, Marchetti GF, Whitney SL (2011) Content comparison of self-report measures used in vestibular rehabilitation based on the international classification of functioning, disability and health. Phys Ther Mar 91(3):346-357

19. Powell LE, Myers AM (1995) The Activities specific Balance Confidence (ABC) scale. J Gerontol A Biol Sci Med Sci 50:28-34

20. Cohen HS, Kimball KT, Adams AS (2000) Application of the vestibular disorders activities of daily living scale. Laryngoscope 110:1204-1209 
21. Duracinsky M, Mosnier I, Bouccara D, Sterkers O, Chassany O (2007) Working Group of the Société Française d'Oto-RhinoLaryngologie (ORL). Literature review of questionnaires assessing vertigo and dizziness, and their impact on patients' quality of life. Value Health 10(4):273-284

22. Gomes Patatas OH, Freitas Gananca C, Freitas Gananca F (2009) Quality of life of individuals submitted to vestibular rehabilitation. Braz J Otorhinolaryngol 75(3):387-394

23. Socher DD, Socher JA, Azzi VJ (2012) Evaluation of quality of life pre- and post-vestibular rehabilitation in patients with benign paroxysmal positional vertigo associated with Meniere's disease. Int Arch Otorhinolaryngol 16(4):430-436

24. Santos EM, Gazzola JM, Gananca CF, Caovilla HH, Gananca FF (2010) Impact of dizziness on the life quality of elderly with chronic vestibulopaty. Pro-Fono 22(4):427-432

25. Nola G, Mostardini C, Salvi V, Ercolani AP, Ralli G (2010) Validity of Italian adaptation of the Dizziness Handicap Inventory (DHI) and evaluation of the quality of life in patients with acute dizziness. Acta Otolaryngologica Italica 30:190-197

26. Fernandez L, Breinbauer HA, Delano PH (2015) Vertigo and dizziness in the elderly. Front Neurol 6:1-6

27. Takano NA, Cavalli SS, Ganança MM, Caovilla HH, Santos MA, Peluso Ede T, Ganança FF (2010) Quality of life in elderly with dizziness. Braz J Otorhinolaryngol 76(6):769-775

28. Lloyd SK, Kasbekar AV, Baguley DM, Moffat DA (2010) Audiovestibular factors influencing quality of life in patients with conservatively managed sporadic vestibular schwannoma. Otol Neurotol 31(6):968-976

29. Lasisi AO, Gureje O (2010) Disability and quality of life among community elderly with dizziness: report from the Ibadan study of ageing. J Laryngol Otol 124(9):957-962

30. Hsu LC, Hu HH, Wong WJ, Wang SJ, Luk YO, Chern CM (2005) Quality of life in elderly patients with dizziness: analysis of the Short-Form Health Survey in 197 patients. Acta Otolaryngol 125(1):55-59

31. Weidt S, Bruehl AB, Straumann D, Hegemann SC, Krautstrunk G, Rufer M (2014) Health-related quality of life and emotional distress in patients with dizziness: a cross-sectional approach to disentangle their relationship. BMC Health Serv Res 22(14):317

32. Soto-Varela A, Huertas-Pardo B, Gayoso-Diz P, Santos-Perez S, Sanchez-Sellero I (2016) Disability perception in Menière's disease: when, how much and why? Eur Arch Otorhinolaryngol 273(4):865-872

33. Mira E (2008) Improving the quality of life in patients with vestibular disorders: the role of medical treatments and physical rehabilitation. Int J Clin Pract 62(1):109-114

34. Ferreira L, Ribeiro K, Costa de Lima K, Diniz J, Guerra RO, Ribeiro AJ, Lima L (2012) Quality of life assessment in elderly with dizziness complain. J Surg Cl Res 3(2):59-67 